\title{
Review of Different Error Metrics: A Case of Solar Forecasting
}

\author{
Pardeep Singla, Manoj Duhan, and Sumit Saroha
}

\begin{abstract}
Renewable energy systems (RES) are no longer confined to being used as a stand-alone entity in the modern era. These RES, especially solar panels are also used with the grid power systems to supply electricity. However, precise forecasting of solar irradiance is necessary to ensure that the grid operates in a balanced and planned manner. Various solar forecasting models (SFM) are presented in the literature to produce an accurate solar forecast. Nevertheless, each model has gone through the step of evaluation of its accuracy using some error measures. Many error measures are discussed in the literature for deterministic as well as probabilistic solar forecasting. But each study has its own selected error measure which sometimes landed on a wrong interpretation of results if not selected appropriately. As a result, this paper offers a critical assessment of several common error metrics with the goal of discussing alternative error metrics and establishing a viable set of error metrics for deterministic and probabilistic solar forecasting. Based on highly cited research from the last three years (20212019), error measures for both types of forecasting are presented with their basic functionalities, advantages \& limitations which equipped the reader to pick the required compatible metrics
\end{abstract}

Index Terms-Error measures, deterministic forecasting, probabilistic forecasting, solar forecasting, root mean square error.

\section{INTRODUCTION}

A mong various renewable energy resources (RES), solar energy has been recognized as one of the potential solutions to the electricity demand [1]. However, the intermittent and uncertain behavior of solar photovoltaic (PV) output is one of the biggest challenges to the grid integrated power systems. As a result, solar forecasting or PV power forecasting (commonly both referred to as solar forecasting) has received unprecedented attention from the various communities of researchers. Progress in solar forecasting has been steadily increasing since the end of the nineteenth century, with the goal of providing accurate solar forecasting models (SFM) [2]. While searching the publications for the keyword "solar forecasting" and "PV forecasting", Google

Pardeep Singla, Research Scholar, Department of ECE, Deenbandhu Chhotu Ram University of Science \& Technology, Sonipat, Haryana, India (email: pradeepsingla7@gmail.com). ORCID:0000-0002-9304-0137

Manoj Duhan, Professor, Department of ECE, Deenbandhu Chhotu Ram University of Science \& Technology, Sonipat, Haryana, India (email: duhan_manoj@rediffmail.com). ORCID: 0000-0003-2745-3053

Sumit Saroha, Assistant Professor, Department of EE, Guru Jambheshwar University of Science \& Technology, Hisar, Haryana, India (email: ssumitsaroha8@gmail.com). ORCID: 0000-0003-2459-6710 scholar (a reputed technical research database) returns 19300 and 16000 results respectively since the year 2020 as on date of $17^{\text {th }}$ August 2021, 11:58 p.m.

From consideration of this abundant literature of solar forecasting, numerous new SFM has been developed and also being developed. Many researchers have also been presented an extensive review on various SFMs. Table 1 presented the top 10 review studies on the SFMs published in recent years. These papers are selected from the year 2019-2021 based on the number of citations from google scholar (as on date $17^{\text {th }}$ Aug., 2021).

The publications mentioned in table 1 only presented the details of different forecasting models and their fundamental methodologies. Nevertheless, these studies are based on a limited number of papers and can only provide a brief of recent works among total selected publications. In other words, these publications act like local optima instead of a global solution in an optimization problem. Moreover, these publications aware the researcher's community about the recent development in the solar forecasting models only.

In parallel, to estimate the performance of any SFM, some statistical measures are adopted by the developers. In the last two decades, various accuracy measures or error measures have been used by developers to evaluate their models. These measures provide the necessary feedback to the decisionmaker for refining and calibrating the measured model to optimize the preciseness of the model. Upon searching the abundant literature of solar forecasting/PV forecasting, no universal or single error metrics has been found that has been accepted by every researcher to evaluate their model. Each study has its own adopted statistical measure on the unexplained ground to prove its accuracy best. Sometimes in practical problems, some of the popular metrics are failed to provide easily interpretable results. For instance, the mean absolute percentage error (MAPE) is one of the popular metrics to evaluate any SFM, but is vulnerable to the outliers [3]. Lastly, despite of developments of various SFMs, the solution of universal single error metrics for all models is still controversial.

TABLE I

RECENTLY PUBLISHED LITERATURE REVIEW ON SOLAR/ PV FORECASTING

\begin{tabular}{lll}
\hline Ref. & YOP & Title \\
\hline$[1]$ & 2019 & A review of deep learning for renewable energy forecasting \\
{$[4]$} & 2019 & $\begin{array}{l}\text { Modeling of solar energy systems using artificial neural } \\
\text { network: A comprehensive review }\end{array}$ \\
\hline
\end{tabular}




\begin{tabular}{|c|c|c|}
\hline [5] & 2019 & $\begin{array}{l}\text { Review on forecasting of photovoltaic power generation based } \\
\text { on machine learning and met heuristic techniques }\end{array}$ \\
\hline [6] & 2020 & $\begin{array}{l}\text { A review and evaluation of the state-of-the-art in PV solar } \\
\text { power forecasting: Techniques and optimization }\end{array}$ \\
\hline [2] & 2019 & $\begin{array}{l}\text { A current perspective on the accuracy of incoming solar } \\
\text { energy forecasting }\end{array}$ \\
\hline [7] & 2019 & $\begin{array}{l}\text { Sustainability perspectives- a review for solar photovoltaic } \\
\text { trends and growth opportunities }\end{array}$ \\
\hline [8] & 2019 & $\begin{array}{l}\text { Clear sky solar irradiance models: A review of seventy } \\
\text { models }\end{array}$ \\
\hline [9] & 2020 & $\begin{array}{l}\text { Advanced Methods for photovoltaic output power forecasting: } \\
\text { A review }\end{array}$ \\
\hline [10] & 2020 & $\begin{array}{l}\text { A comprehensive review of hybrid models for solar radiation } \\
\text { forecasting }\end{array}$ \\
\hline [11] & 2020 & $\begin{array}{l}\text { Solar irradiance measurement instrumentation and power } \\
\text { solar generation forecasting based on Artificial Neural } \\
\text { Networks (ANN): A review of five years research trend }\end{array}$ \\
\hline
\end{tabular}

Based on the above ground, this paper provides a review of various popular error measures for the case of solar forecasting/ PV forecasting. This paper discusses the different error measures used in deterministic as well as probabilistic forecasting. The critical findings from various studies about the error measures are thoroughly discussed in the presented manuscript.

This paper is organized is as follows: section 2 discusses the different error measures for the deterministic and probabilistic solar forecasting. Section 3 provides the critical analysis of the error measures used by recent studies. The key findings are also discussed in this section. Section 4 presents some cautions about referring the previous work and section 5 conclude the paper.

\section{ERROR MEASURES}

By examining the recent literature of solar forecasting, the numerous error measures have been obtained. All the measures can be categories for the type of forecasting: deterministic solar forecasting and probabilistic solar forecasting. Fig. 1 shows the two types of solar forecasting along with their respective error measures.

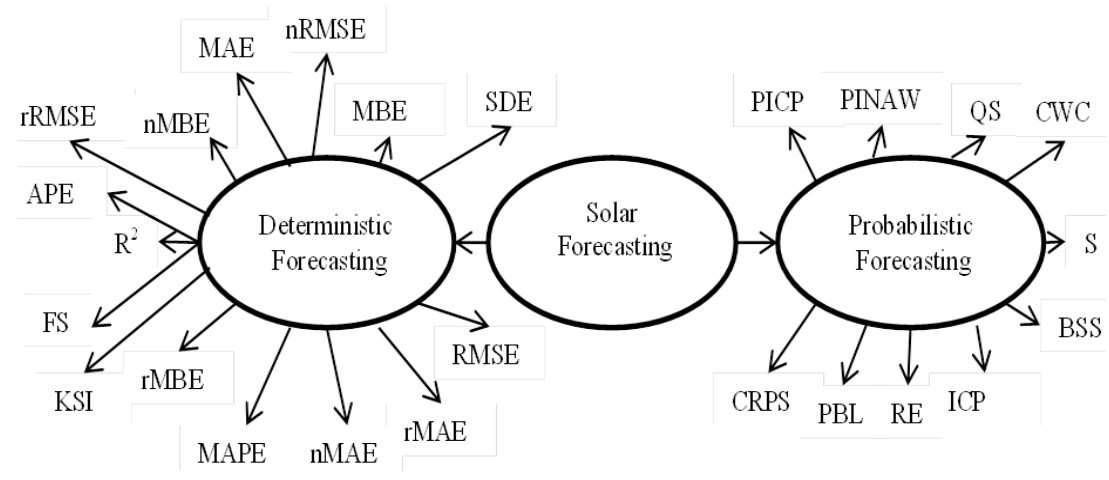

Fig.1. Different errors used in solar forecasting

\section{A. Error Measures of deterministic forecasting}

If $\mathrm{M}$ is the total samples of solar/ $\mathrm{PV}$ data series, $F_{t}$ is the original solar/ $\mathrm{PV}$ series and $\widehat{F}_{t}$ is the forecasted solar /PV series at any time stamp $\mathrm{t}$ then error measures can be represented mathematically as [3]:

Mean Bias Error (MBE): $\mathrm{MBE}$ is used to calculate the average bias in the forecast.

$$
M B E=\frac{1}{M} \sum_{t=1}^{M}\left(\hat{F}_{t}-F_{t}\right)
$$

The value of MBE in positive direction represents the overestimation by the SFM whereas, negative value represents the underestimation.

Mean Absolute Error (MAE): A uniform estimation of error is identified by this measure.

$M A E=\frac{1}{M} \sum_{t=1}^{M}\left|\widehat{F}_{t}-F_{t}\right|$

Standard Deviation Error (SDE): This measure used to estimate the deviations from the mean.

$$
S D E=\sqrt{\frac{1}{M} \sum_{t=1}^{M}\left(\widehat{F}_{t}-F_{t}-M B E\right)^{2}}
$$

Root Mean Square Error: It is one of the popular error measures used to evaluate the performance of SFM. This measure identifies the largest error in the forecasted sequence.

$$
\begin{aligned}
& R M S E=\sqrt{\frac{1}{M} \sum_{t=1}^{M}\left(\widehat{F}_{t}-F_{t}\right)^{2}} \\
& R M S E^{2}=M B E^{2}+S D E^{2}
\end{aligned}
$$

Mean Absolute Percentage Error (MAPE): This is simply the representation of uniform error (MAE) in percentage form.

$$
M A P E=\frac{1}{M} \sum_{t=1}^{M}\left|\frac{\widehat{F}_{t}-F_{t}}{F_{t}}\right|
$$

Normalized RMSE: It is RMSE on a different scale. It can be calculated from RMSE of the forecasted output to the mean of forecasted data.

$$
n R M S E=\frac{\sqrt{\frac{1}{M} \sum_{t=1}^{M}\left(\widehat{F}_{t}-F_{t}\right)^{2}}}{\operatorname{mean}\left(\hat{F}_{t}\right)}
$$

Normalized MBE: It is MBE on a different scale. It can be calculated from MBE of the forecasted output to the mean of forecasted data.

$n M B E=\frac{M B E}{\operatorname{mean}\left(\hat{F}_{t}\right)}$ 
Normalized MAE: It is MAE on a different scale. It can be calculated from MAE of the forecasted output to the mean of forecasted data.

$$
n M A E=\frac{M A E}{\operatorname{mean}\left(\hat{F}_{t}\right)}
$$

Forecast Skill: It is a unit less measure to evaluate the effectiveness of the SFM. It can be computed with reference to any benchmark model in term of RMSE, MAPE, and MAE etc.

For a case of RMSE, $F S=1-\frac{R M S E_{\text {observed }}}{R M S E_{\text {ref }}}$

Kolonogorov-Smirnov test Integral (KSI): It is another notable error measure in case of point or deterministic solar forecasting. Unlike other error measure, it compares the cumulative distribution function $(\mathrm{CDF})$ of the forecasted series and the actual series. It can be represented as:

$$
K S I=\int_{x_{\min }}^{x_{\max }} K_{n} d x
$$

Where $\mathrm{x}_{\min } \& \mathrm{x}_{\max }$ are the minimum and maximum values from the forecasted data, $K_{n}=$ Difference in the two CDFs.

A zero value of KSI interpreted as the equal CDF of both series.

Correlation Coefficient: It represents the strength in the linear relationship between the forecasted values and the observed values.

$$
R=\frac{\sum_{t=0}^{M}\left(\hat{F}_{t}-\operatorname{avg}\left(\hat{F}_{t}\right)\right)\left(F_{t}-\operatorname{avg}\left(F_{t}\right)\right)}{\sqrt{\sum_{t=0}^{M}\left(\hat{F}_{t}-\operatorname{avg}\left(\hat{F}_{t}\right)\right)^{2}} \sqrt{\sum_{t=0}^{M}\left(F_{t}-\operatorname{avg}\left(F_{t}\right)\right)^{2}}}
$$

Table II represents the different error measure applications by the various highly cited studies in the recent years to observe the performance of the SFM. The used measure is denoted by the symbol of tick against each paper. However, the Colum "other" comprises of small error measures like SDE, Bias, skewness, kurtosis etc.

\begin{tabular}{|c|c|c|c|c|c|c|c|c|c|c|c|}
\hline Ref. & YOP & RMSE & MAPE & MAE & MBE & nRMSE & nMAE & nMBE & FS & $\mathrm{R}^{2}$ & Others \\
\hline [12] & 2021 & & & & & $\checkmark$ & $\checkmark$ & $\checkmark$ & $\checkmark$ & & $\checkmark$ \\
\hline [13] & 2021 & $\checkmark$ & & & $\checkmark$ & & & & $\checkmark$ & & \\
\hline [14] & 2021 & & & & & & & & & & \\
\hline [15] & 2021 & $\checkmark$ & & $\checkmark$ & $\checkmark$ & & & & $\checkmark$ & & \\
\hline [16] & 2021 & $\checkmark$ & & $\checkmark$ & & & & & & $\checkmark$ & $\checkmark$ \\
\hline [17] & 2021 & $\checkmark$ & & $\checkmark$ & & & & & $\checkmark$ & $\checkmark$ & $\checkmark$ \\
\hline [18] & 2021 & $\checkmark$ & & $\checkmark$ & & & & & & $\checkmark$ & \\
\hline [19] & 2021 & $\checkmark$ & & $\checkmark$ & & & & & & $\checkmark$ & \\
\hline [20] & 2021 & $\checkmark$ & & & & & & & & & $\checkmark$ \\
\hline [21] & 2021 & & $\checkmark$ & & & & & & & & $\checkmark$ \\
\hline [22] & 2020 & $\checkmark$ & $\checkmark$ & & $\checkmark$ & & & & & & \\
\hline [23] & 2020 & $\checkmark$ & & $\checkmark$ & & & & & & & $\checkmark$ \\
\hline [24] & 2020 & $\checkmark$ & & & & & & & & $\checkmark$ & \\
\hline [25] & 2020 & $\checkmark$ & $\checkmark$ & & $\checkmark$ & & & & & & \\
\hline [26] & 2020 & $\checkmark$ & $\checkmark$ & & & $\checkmark$ & & & & $\checkmark$ & \\
\hline [27] & 2020 & $\checkmark$ & $\checkmark$ & & & & & & & $\checkmark$ & \\
\hline [28] & 2020 & & & & & $\checkmark$ & $\checkmark$ & & & & \\
\hline [29] & 2020 & $\checkmark$ & & $\checkmark$ & & $\checkmark$ & $\checkmark$ & & & $\checkmark$ & \\
\hline [30] & 2020 & $\checkmark$ & & & & & & $\checkmark$ & & $\checkmark$ & \\
\hline [31] & 2020 & & $\checkmark$ & & & $\checkmark$ & & & $\checkmark$ & & \\
\hline [32] & 2019 & $\checkmark$ & & & & & & & & & \\
\hline [33] & 2019 & $\checkmark$ & & $\checkmark$ & & $\checkmark$ & $\checkmark$ & & & & \\
\hline [34] & 2019 & $\checkmark$ & $\checkmark$ & $\checkmark$ & & & & & & & \\
\hline [35] & 2019 & $\checkmark$ & $\checkmark$ & $\checkmark$ & & & & & & & \\
\hline [36] & 2019 & & $\checkmark$ & & & & & & & & $\checkmark$ \\
\hline [37] & 2019 & & & & & $\checkmark$ & & $\checkmark$ & $\checkmark$ & & \\
\hline [38] & 2019 & $\checkmark$ & & & & & & & & $\checkmark$ & \\
\hline [39] & 2019 & $\checkmark$ & & $\checkmark$ & & & & & & $\checkmark$ & \\
\hline [40] & 2019 & $\checkmark$ & $\checkmark$ & $\checkmark$ & & & & & & & $\checkmark$ \\
\hline [41] & 2019 & & $\checkmark$ & $\checkmark$ & & $\checkmark$ & $\checkmark$ & & & & $\checkmark$ \\
\hline
\end{tabular}

TABLE II

ERROR MEASURES OF DETERMINISTIC SOLAR FORECASTING IN RECENT PUBLICATIONS

\section{B. Error measures of probabilistic forecasting}

Prediction interval nominal confidence (PINC): The prediction interval (PI) is one of the prime parameters in the probabilistic forecasting which depicts about the probability of lying forecasting values within any specified range. In parallel, the PI directly affected by the value of significance level $\alpha$. So, PINC is the calculation of probability for the future value of solar output that falls within the PI.

$$
P I N C=100(1-\alpha) \%
$$

In addition, the PI for a time $\mathrm{t}$ and significance level $\alpha$ can be represented as: where $\mathrm{U}_{\mathrm{L}} \& \mathrm{~L}_{\mathrm{t}}$ are the upper and lower level of PI respectively.

Prediction interval coverage probability (PICP): it is used to evaluate the distributions of the forecasted values and can be expressed as:

$$
P I C P=\frac{1}{M} \sum_{t=0}^{n} C_{t}
$$

where $M$ is the total sample in the observations and $C_{t}$ is 
$C_{t}= \begin{cases}1, & \text { if } \hat{F}_{t} \in P_{t} \\ 0, & \text { otherwise }\end{cases}$

Prediction interval normalized average width (PINAW):

PINAW $=\frac{1}{M R} \sum_{t=0}^{n} P_{t}$

where $\mathrm{R}$ is the range of observed values.

Coverage width-based criterion (CWC):

This measure used the values of PICP and PINAW to evaluate the SFM.

$C W C=P I N A W\left[1+\beta(P I C P) e^{-\gamma(P I C P-\varepsilon)}\right]$

where $\beta$ (PICP) is a step function that can be represented as:

$\beta(P I C P)= \begin{cases}0, & \text { if } P I C P \geq \varepsilon \\ 1, & \text { if } P I C P<\varepsilon\end{cases}$
Continuous ranked probability score (CRPS): This is one of most widely used error measure in case of probabilistic forecasting. Like MAE in deterministic forecasting, it also generalizes in MAE for the probabilistic forecasting.

$$
C R P S=\frac{1}{M} \sum_{t=0}^{M} \int_{0}^{P}\left(C D F_{t}-\eta\left(F_{t}-\hat{F}_{t}\right)\right)^{2} d F
$$

where $\eta$ is the Heaviside step function represented as:

$\eta= \begin{cases}0 & \text { if } F_{t}<\hat{F}_{t} \\ 1 & \text { otherwise }\end{cases}$

To analyze the popularity of each probabilistic error measure, table III has been prepared from the highly cited publication of recent years.

TABLE III

ERROR MEASURES OF PROBABILISTIC SOLAR FORECASTING IN RECENT PUBLICATIONS

\begin{tabular}{|c|c|c|c|c|c|c|c|c|c|c|c|c|}
\hline Ref. & YOP & CRPS & $\mathrm{RE}$ & S & PICP & PINAW & BSS & CRPSS & $\mathrm{QS}$ & CWC & PBL & Other \\
\hline [42] & 2021 & $\checkmark$ & $\checkmark$ & & $\checkmark$ & & & & & & & $\checkmark$ \\
\hline [43] & 2021 & $\checkmark$ & $\checkmark$ & & & & $\checkmark$ & $\checkmark$ & & & & \\
\hline [44] & 2021 & $\checkmark$ & & & & & & $\checkmark$ & & & & \\
\hline [45] & 2021 & $\checkmark$ & & & & & & $\checkmark$ & $\checkmark$ & & & \\
\hline [46] & 2021 & & & & $\checkmark$ & $\checkmark$ & & & & & & $\checkmark$ \\
\hline [47] & 2021 & $\checkmark$ & & & & & & $\checkmark$ & & & & \\
\hline [48] & 2021 & $\checkmark$ & $\checkmark$ & $\checkmark$ & & & & & & & & \\
\hline [49] & 2020 & $\checkmark$ & & & $\checkmark$ & $\checkmark$ & & & & & & \\
\hline [50] & 2020 & $\checkmark$ & $\checkmark$ & $\checkmark$ & & $\checkmark$ & & & & & & \\
\hline [51] & 2020 & & & $\checkmark$ & & & & & & & & $\checkmark$ \\
\hline [52] & 2020 & & & & $\checkmark$ & $\checkmark$ & & & & & & \\
\hline [53] & 2020 & $\checkmark$ & & & & & $\checkmark$ & & & & & $\checkmark$ \\
\hline [54] & 2020 & $\checkmark$ & $\checkmark$ & & & $\checkmark$ & & $\checkmark$ & & & & $\checkmark$ \\
\hline [55] & 2020 & $\checkmark$ & & & & $\checkmark$ & & & & & & \\
\hline [56] & 2020 & & $\checkmark$ & & $\checkmark$ & $\checkmark$ & & $\checkmark$ & & & $\checkmark$ & \\
\hline [57] & 2020 & $\checkmark$ & & & $\checkmark$ & & & $\checkmark$ & $\checkmark$ & & & \\
\hline [58] & 2020 & $\checkmark$ & & & $\checkmark$ & $\checkmark$ & & & & & $\checkmark$ & \\
\hline [59] & 2020 & & $\checkmark$ & $\checkmark$ & & & & & & & $\checkmark$ & \\
\hline [60] & 2020 & $\checkmark$ & & & & & & $\checkmark$ & & & & \\
\hline [61] & 2020 & & & & $\checkmark$ & $\checkmark$ & & & & $\checkmark$ & & \\
\hline [62] & 2020 & & & & $\checkmark$ & & & & & & & $\checkmark$ \\
\hline [63] & 2020 & $\checkmark$ & $\checkmark$ & & & & & & & & & \\
\hline [64] & 2019 & $\checkmark$ & & & & $\checkmark$ & & $\checkmark$ & & & & \\
\hline [65] & 2019 & & & & & $\checkmark$ & & & & $\checkmark$ & & \\
\hline [66] & 2019 & $\checkmark$ & & & & & $\checkmark$ & & & & & $\checkmark$ \\
\hline [67] & 2019 & & & & & & & & & & & \\
\hline
\end{tabular}

In table III, each error measure according to their application in respective study is mark with the symbol "tick". The other matrices represent the average coverage error (ACE), barrier score (BS) etc. In addition, based on these references, the percentage share of each error metrices in deterministic and probabilistic forecasting is shown in fig. 2. It is evident from the fig. 2(a), RMSE has greatest share among all error metrices, represents the first choice of researchers to represents the forecasting accuracy of their models. Whereas, the MAPE \& MAE scored with the same percentage. Similarly, as can be seen in fig. 2(b), the CRPS and PINAW are the mostly used error metrices for the evaluation of probabilistic forecasting.

\section{CRITICAL REVIEW}

This section provides the critical findings of the entire survey of the error measures. From the critical examinations of several measures, a question arises that what is the ideal error measure? Some studies suggested a measures, that are capable of interpret the results easily and sensitive to the outliers i.e., robustness. On the other hand, some have been suggested that the criteria of forecast evaluation must correspond to the criteria of forecast optimization. While some are in favour of the scale independent error measures. Therefore, the following are the critical observations on the popular error measure that have been used by most cited papers as mentioned in table II \& III. 


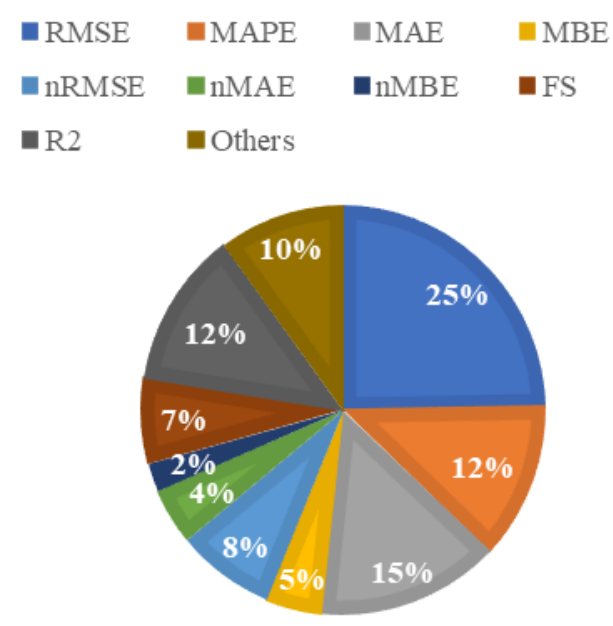

Fig. 2 (a) Percentage share of different error metrices in deterministic forecasting.

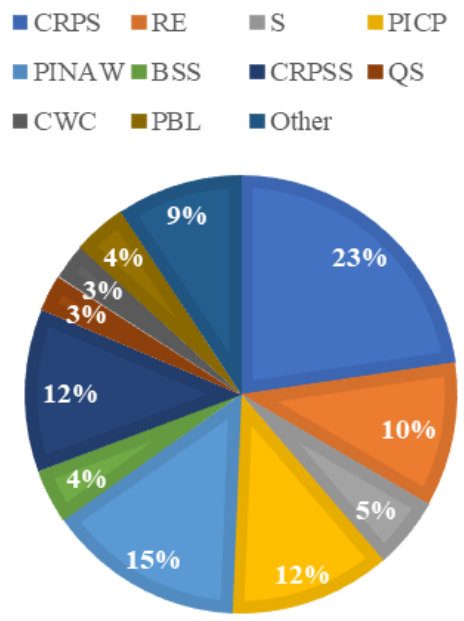

Fig. 2 (b) Percentage share of different error metrices in probabilistic forecasting.

\section{A. Key findings: Deterministic forecasting}

It is apparent from table II and fig. 2 (a), the RMSE is most popular and common error measure used to evaluate the SFM. It is widely used to observe the model performance in case of determining solar forecast. It is due to the fact that the largest errors in the solar forecasting are highly undesirable. RMSE penalize the model performance for the highest errors present in the final results [11].

MAE and MBE are the second most widely used error measure after RMSE for the evaluation of SFM. However, they are scale dependent measures and can show biased results for different scales. These error measures are only useful in case where the results achieve the Gaussian distribution [69].

MAPE is a highly unsuitable error measure for examination of the accuracy of the SFM. The results of this measure are highly affected by skewed and diffused distributions. The interpretation of MAPE results also becomes inefficient in large skewed distributions due to the high influence of outliers. Moreover, the MAPE penalizes the positive values of error instead of negative values which also leads to biased results [3]. However, many of the authors mentioned in table 2 used this measure to shows the average errors in percentage but statistically; it is a poor measure due to non-symmetric losses and extreme percentages.

MASE is used to overcome the problem of MAPE, MASE is considered to evaluate the performance of a SFM. It is another MAE but scaled by the MAE of benchmark/ reference model [11]. However, MAAPE; another version of MAPE is uncommon in the literature of solar forecasting but also used by some of the potential studies [70]. It transforms the MAPE into MAAPE measure using the arctangent function. This measure not only removes the shortcomings of the MAPE but also preserve the original characteristics of the MAPE. Therefore, MAAPE could be a good statistical error measure for observing the performance of SFM instead of MAPE.

KSI is another notable error measure to evaluate the accuracy of the point forecast of solar irradiance. This measure never compares the forecasted values of a SFM to its respective actual values as in other measures. Instead, a gathering of forecast distribution of the SFM makes it more popular. Furthermore, it is popularly used in the case of time series-based forecasting where its emphasis is on the variability of forecasted values with respect to actual values. In other words, by using this error measure, the lesser variability in the forecast can be detected by obtaining the larger value of KSI [11].

\section{B. Key findings: Probabilistic forecasting}

Apart from point forecast, probabilistic forecast produces highly satisfactory results in solar forecasting. Point forecast provides a fixed forecast at a specific interval of time. Whereas, probabilistic forecast provides the detail of uncertainty in forecasted results using a range of expectations in terms of prediction interval (PI), where the forecast value will fall. In other words, PI forecasting generates to achieve a density function of the desired value.

PICP, PINAW are the two most commonly used parameters to observe the reliability of a PI. However, PICP determines the probability of the forecast fall within the range. In parallel, PINAW refers to the width of the PI. Therefore, these two are conflicting measures as both are required either high or low in a good forecast [42]. Another most common measures in probabilistic forecasting are $\mathrm{CWC}$, which is the combination of PICP and PINAW. The main point in the CWC is that it penalizes the invalid PIs which indirectly enforce the good forecast to achieve valid PICP. Lastly, CRPS is global measure in the probabilistic forecasting and can be understood as MAE in deterministic forecasting. Unlike other metrics, the CRPS is calculated by determining the cumulative distribution function $(\mathrm{CDF})$ of the forecast. So, probabilistic forecasting is, however, a better forecasting method but observed lesser work on it compared to deterministic forecasting.

\section{CAUTIONS}

While working with solar forecasting, either in deterministic forecasting or probabilistic forecast, it is necessary to use the terms cautiously. With the huge literature on deterministic forecasting, many of the studies altered the universal naming conventions as well as used different 
acronyms for a known error measure. For instance, Gueymard et al. (2014) obtained the percentage value of RMSE, MAE and MBE but the acronyms of error metrics were not changed [68]. Likewise, the word "deviation" was used many times instead of the actual word "error". Moreover, a normalization of any error measure can be obtained by dividing the mean of the sample. But sometimes, to represents the lesser values of normalized error measures, studies divided it by the maximum value from the sample [69].

\section{CONCLUSION}

While developing a solar forecasting model, a clear understanding of the error measures is highly desirable. Since, an unclear and inappropriate selection of the error measure leads to a different conclusion. This paper is designed with an aim to present a clear representation of different error measures of deterministic and probabilistic forecasting in solar/ PV forecasting. Various key findings are obtained from the highly cited studies of the latest literature. From this study of highly cited papers from latest literature (year 2021-2019), it is found that the RMSE, MAE, MAPE and $\mathrm{R}^{2}$ are the most common error metrics in deterministic forecasting with the $25 \%, 15 \%, 12 \%$ and $12 \%$ respectively. On the other hand, CRPS, PINAW, PICP and RE are the most common error metrics in probabilistic forecasting with $23 \%, 15 \%, 12 \%$ and $10 \%$ respectively share among all. Conclusively, it is observed that the performance of a solar forecasting model is highly dependent on the type of geographical area as well as the type of weather. Therefore, a scale-dependent error measure like "forecast skill" is advised to compare the performance of the different models for different datasets. Therefore, for a solar forecasting model, the discussed error measures are recommended in practice. However, for a future prospectus, a new error measure can be developed with the characteristics of scale independence and free from bias.

\section{ABBREVIATIONS}

APE: Absolute percentage error; BSS: Brier skill score; CRPS: Continuous ranked probability score; CWC: Coverage width-based criterion; FS: Forecast skill; ICP: Interval coverage probability; KSI: Kolonogorov-Smirnov test Integral; MAE: Mean absolute error; MAPE; Mean absolute percentage error; MBE: Mean bias error; PINAW: Prediction interval normalized average width; PICP: Prediction interval coverage probability; PINC: Prediction interval nominal confidence; QS: Quantile score; RMSE: Root mean square error; rMAE: relative mean absolute error; rMBE: Relative mean bias error; rRMSE: relative root mean square error; R2: Coefficient of determination; RE: reliability; S: sensitivity; SDE: standard deviation error;

\section{REFERENCES}

H. Wang, Z. Lei, X. Zhang, B. Zhou, and J. Peng, "A review of deep learning for renewable energy forecasting," Energy Conversion and Management, vol. 198. Elsevier Ltd, p. 111799, Oct. 15, 2019, doi: 10.1016/j.enconman.2019.111799.

[2] R. Blaga, A. Sabadus, N. Stefu, C. Dughir, M. Paulescu, and V. Badescu, "A current perspective on the accuracy of incoming solar energy forecasting," Prog. Energy Combust. Sci., vol. 70, pp. 119-
144, Jan. 2019, doi: 10.1016/J.PECS.2018.10.003.

P. Singla, M. Duhan, and S. Saroha, "An ensemble method to forecast 24-h ahead solar irradiance using wavelet decomposition and BiLSTM deep learning network," Earth Sci Inform, pp. 1-16, Nov. 2021, doi: https://doi.org/10.1007/s12145-021-00723-1.

[4] M. N. Akhter, S. Mekhilef, H. Mokhlis, and N. M. Shah, "Review on forecasting of photovoltaic power generation based on machine learning and metaheuristic techniques," IET Renew. Power Gener., vol. 13, no. 7, pp. 1009-1023, May 2019, doi: 10.1049/IETRPG.2018.5649.

[5] R. Ahmed, V. Sreeram, Y. Mishra, and M. D. Arif, "A review and evaluation of the state-of-the-art in PV solar power forecasting: Techniques and optimization," Renew. Sustain. Energy Rev., vol. 124, no. June 2019, p. 109792, 2020, doi: 10.1016/j.rser.2020.109792.

[6] P. Choudhary and R. K. Srivastava, "Sustainability perspectives- a review for solar photovoltaic trends and growth opportunities," J. Clean. Prod., vol. 227, pp. 589-612, Aug. 2019, doi: 10.1016/J.JCLEPRO.2019.04.107.

[7] F. Antonanzas-Torres, R. Urraca, J. Polo, O. Perpiñán-Lamigueiro, and R. Escobar, "Clear sky solar irradiance models: A review of seventy models," Renew. Sustain. Energy Rev., vol. 107, pp. 374387, Jun. 2019, doi: 10.1016/J.RSER.2019.02.032.

[8] A. Mellit, A. M. Pavan, E. Ogliari, S. Leva, and V. Lughi, "Advanced methods for photovoltaic output power forecasting: A review," Appl. Sci., vol. 10, no. 2, p. 487, 2020, doi: 10.3390/app10020487.

[9] M. Guermoui, F. Melgani, K. Gairaa, and M. L. Mekhalfi, "A comprehensive review of hybrid models for solar radiation forecasting," J. Clean. Prod., vol. 258, p. 120357, 2020, doi: 10.1016/j.jclepro.2020.120357.

[10] A. R. Pazikadin, D. Rifai, K. Ali, M. Z. Malik, A. N. Abdalla, and M. A. Faraj, "Solar irradiance measurement instrumentation and power solar generation forecasting based on Artificial Neural Networks (ANN): A review of five years research trend," Sci. Total Environ., vol. 715, p. 136848, May 2020, doi: 10.1016/J.SCITOTENV.2020.136848.

[11] P. Singla, M. Duhan, and S. Saroha, "A comprehensive review and analysis of solar forecasting techniques," Front. Energy, pp. 1-37, Mar. 2021, doi: 10.1007/s11708-021-0722-7.

[12] M. J. Mayer and G. Gróf, "Extensive comparison of physical models for photovoltaic power forecasting," Appl. Energy, vol. 283, p. 116239, Feb. 2021, doi: 10.1016/j.apenergy.2020.116239.

[13] P. Kumari and D. Toshniwal, "Extreme gradient boosting and deep neural network based ensemble learning approach to forecast hourly solar irradiance," J. Clean. Prod., vol. 279, p. 123285, Jan. 2021, doi: 10.1016/j.jclepro.2020.123285.

[14] M. Guermoui, K. Gairaa, J. Boland, and T. Arrif, "A Novel Hybrid Model for Solar Radiation Forecasting Using Support Vector Machine and Bee Colony Optimization Algorithm: Review and Case Study," J. Sol. Energy Eng., vol. 143, no. 2, Apr. 2021, doi: $10.1115 / 1.4047852$.

[15] S. Das, "Short term forecasting of solar radiation and power output of 89.6kWp solar PV power plant," Mater. Today Proc., vol. 39, pp. 1959-1969, Jan. 2021, doi: 10.1016/J.MATPR.2020.08.449.

[16] E. Jumin, F. B. Basaruddin, Y. B. M. Yusoff, S. D. Latif, and A. N. Ahmed, "Solar radiation prediction using boosted decision tree regression model: A case study in Malaysia," Environ. Sci. Pollut. Res. 2021 2821, vol. 28, no. 21, pp. 26571-26583, Jan. 2021, doi: 10.1007/S11356-021-12435-6.

[17] P. Kumari and D. Toshniwal, "Long short term memoryconvolutional neural network based deep hybrid approach for solar irradiance forecasting," Appl. Energy, vol. 295, p. 117061, Aug. 2021, doi: 10.1016/J.APENERGY.2021.117061.

[18] G. Narvaez, L. F. Giraldo, M. Bressan, and A. Pantoja, "Machine learning for site-adaptation and solar radiation forecasting," Renew. Energy, vol. 167, pp. 333-342, Apr. 2021, doi: 10.1016/J.RENENE.2020.11.089.

[19] T. Peng, C. Zhang, J. Zhou, and M. S. Nazir, "An integrated framework of Bi-directional long-short term memory (BiLSTM) based on sine cosine algorithm for hourly solar radiation forecasting," Energy, vol. 221, p. 119887, Apr. 2021, doi: 10.1016/j.energy.2021.119887.

[20] B. K. Puah et al., "A regression unsupervised incremental learning algorithm for solar irradiance prediction,” Renew. Energy, vol. 164, 
Pp. 908-925, Feb. 2021, doi. 10.1016/J.RENENE.2020.09.080 M. O. Moreira, P. P. Balestrassi, A. P. Paiva, P. F. Ribeiro, and B. D. Bonatto, "Design of experiments using artificial neural network ensemble for photovoltaic generation forecasting," Renew. Sustain. Energy Rev., vol. 135, p. 110450, Jan. 2021, doi: 10.1016/J.RSER.2020.110450.

[22] P. Li, K. Zhou, X. Lu, and S. Yang, "A hybrid deep learning model for short-term PV power forecasting," Appl. Energy, vol. 259, p. 114216, Feb. 2020, doi: 10.1016/j.apenergy.2019.114216.

[23] H. Zang, L. Cheng, T. Ding, K. W. Cheung, Z. Wei, and G. Sun, "Day-ahead photovoltaic power forecasting approach based on deep convolutional neural networks and meta learning," Int. J. Electr. Power Energy Syst., vol. 118, p. 105790, Jun. 2020, doi: 10.1016/j.ijepes.2019.105790.

[24] H. Sharadga, S. Hajimirza, and R. S. Balog, "Time series forecasting of solar power generation for large-scale photovoltaic plants," Renew. Energy, vol. 150, pp. 797-807, May 2020, doi: 10.1016/j.renene.2019.12.131

[25] F. Wang et al., "A minutely solar irradiance forecasting method based on real-time sky image-irradiance mapping model," Energy Convers. Manag., vol. 220, p. 113075, Sep. 2020, doi: 10.1016/J.ENCONMAN.2020.113075.

[26] Y. Jung, J. Jung, B. Kim, and S. U. Han, "Long short-term memory recurrent neural network for modeling temporal patterns in longterm power forecasting for solar PV facilities: Case study of South Korea,” J. Clean. Prod., vol. 250, p. 119476, Mar. 2020, doi: 10.1016/J.JCLEPRO.2019.119476.

[27] J. Lee, W. Wang, F. Harrou, and Y. Sun, "Reliable solar irradiance prediction using ensemble learning-based models: A comparative study," Energy Convers. Manag., vol. 208, p. 112582, Mar. 2020, doi: 10.1016/J.ENCONMAN.2020.112582.

[28] M. AlKandari and I. Ahmad, "Solar power generation forecasting using ensemble approach based on deep learning and statistical methods," Appl. Comput. Informatics, no. xxxx, 2019, doi: 10.1016/j.aci.2019.11.002

[29] H. Zang, L. Liu, L. Sun, L. Cheng, Z. Wei, and G. Sun, "Short-term global horizontal irradiance forecasting based on a hybrid CNNLSTM model with spatiotemporal correlations," Renew. Energy, vol. 160, pp. 26-41, Nov. 2020, doi: 10.1016/j.renene.2020.05.150.

[30] Z. Pang, F. Niu, and Z. O'Neill, “Solar radiation prediction using recurrent neural network and artificial neural network: A case study with comparisons," Renew. Energy, 2020, doi: 10.1016/j.renene.2020.04.042.

[31] S. Theocharides, G. Makrides, A. Livera, M. Theristis, P. Kaimakis, and G. E. Georghiou, "Day-ahead photovoltaic power production forecasting methodology based on machine learning and statistical post-processing,” Appl. Energy, vol. 268, p. 115023, Jun. 2020, doi: 10.1016/J.APENERGY.2020.115023.

[32] M. Abdel-Nasser and K. Mahmoud, "Accurate photovoltaic power forecasting models using deep LSTM-RNN," Neural Comput. Appl., vol. 31, pp. 2727-2740, 2019, doi: https://doi.org/10.1007/s00521-017-3225-z.

[33] L. Benali, G. Notton, A. Fouilloy, C. Voyant, and R. Dizene, "Solar radiation forecasting using artificial neural network and random forest methods: Application to normal beam, horizontal diffuse and global components," Renew. Energy, vol. 132, pp. 871-884, Mar. 2019, doi: 10.1016/j.renene.2018.08.044.

[34] K. Wang, X. Qi, and H. Liu, "A comparison of day-ahead photovoltaic power forecasting models based on deep learning neural network," Appl. Energy, vol. 251, Oct. 2019, doi: 10.1016/j.apenergy.2019.113315.

[35] H. Zhou, Y. Zhang, L. Yang, Q. Liu, K. Yan, and Y. Du, "ShortTerm photovoltaic power forecasting based on long short term memory neural network and attention mechanism," IEEE Access, vol. 7, pp. 78063-78074, 2019, doi: 10.1109/ACCESS.2019.2923006

[36] S. Ghimire, R. C. Deo, N. Raj, and J. Mi, "Deep solar radiation forecasting with convolutional neural network and long short-term memory network algorithms," Appl. Energy, vol. 253, p. 113541, Nov. 2019, doi: 10.1016/j.apenergy.2019.113541.

[37] G. M. Yagli, D. Yang, and D. Srinivasan, "Automatic hourly solar forecasting using machine learning models," Renew. Sustain. Energy Rev., vol. 105, no. October 2018, pp. 487-498, 2019, doi: 10.1016/j.rser.2019.02.006.

[38] M. Alsharif, M. Younes, and J. Kim, "Time Series ARIMA Model for Prediction of Daily and Monthly Average Global Solar Radiation: The Case Study of Seoul, South Korea," Symmetry (Basel)., vol. 11, no. 2, p. 240, Feb. 2019, doi: $10.3390 /$ sym 11020240 .

[39] S. Ghimire, R. C. Deo, N. J. Downs, and N. Raj, "Global solar radiation prediction by ANN integrated with European Centre for medium range weather forecast fields in solar rich cities of Queensland Australia," J. Clean. Prod., vol. 216, pp. 288-310, Apr. 2019, doi: 10.1016/j.jclepro.2019.01.158.

[40] K. Wang, X. Qi, and H. Liu, "Photovoltaic power forecasting based LSTM-Convolutional Network," Energy, vol. 189, p. 116225, Dec. 2019, doi: 10.1016/J.ENERGY.2019.116225.

[41] A. Nespoli et al., "Day-Ahead Photovoltaic Forecasting: A Comparison of the Most Effective Techniques," Energies, vol. 12, no. 9, p. 1621, Apr. 2019, doi: 10.3390/en12091621.

[42] G. Liu et al., "Probabilistic spatiotemporal solar irradiation forecasting using deep ensembles convolutional shared weight long short-term memory network," Appl. Energy, vol. 300, p. 117379, Oct. 2021, doi: 10.1016/J.APENERGY.2021.117379.

[43] J. Le Gal La Salle, M. David, and P. Lauret, "A new climatology reference model to benchmark probabilistic solar forecasts," Sol. Energy, vol. 223, pp. 398-414, Jul. 2021, doi: 10.1016/J.SOLENER.2021.05.037.

[44] B. Schulz, M. El Ayari, S. Lerch, and S. Baran, "Post-processing numerical weather prediction ensembles for probabilistic solar irradiance forecasting," Sol. Energy, vol. 220, pp. 1016-1031, May 2021, doi: 10.1016/J.SOLENER.2021.03.023.

[45] K. Doubleday, S. Jascourt, W. Kleiber, and B. M. Hodge, "Probabilistic solar power forecasting using bayesian model averaging," IEEE Trans. Sustain. Energy, vol. 12, no. 1, pp. 325337, Jan. 2021, doi: 10.1109/TSTE.2020.2993524.

[46] F. Najibi, D. Apostolopoulou, and E. Alonso, "Enhanced performance Gaussian process regression for probabilistic shortterm solar output forecast," Int. J. Electr. Power Energy Syst., vol. 130, p. 106916, Sep. 2021, doi: 10.1016/J.IJEPES.2021.106916.

[47] X. Zhang, F. Fang, and J. Wang, "Probabilistic Solar Irradiation Forecasting Based on Variational Bayesian Inference with Secure Federated Learning," IEEE Trans. Ind. Informatics, vol. 17, no. 11, pp. 7849-7859, Nov. 2021, doi: 10.1109/TII.2020.3035807.

[48] Z. Zhang et al., "Solar Radiation Intensity Probabilistic Forecasting Based on K-Means Time Series Clustering and Gaussian Process Regression,” IEEE Access, vol. 9, pp. 89079-89092, 2021, doi: 10.1109/ACCESS.2021.3077475.

[49] G. M. Yagli, D. Yang, and D. Srinivasan, "Reconciling solar forecasts: Probabilistic forecasting with homoscedastic Gaussian errors on a geographical hierarchy," Sol. Energy, vol. 210, pp. 5967, Nov. 2020, doi: 10.1016/J.SOLENER.2020.06.005.

[50] M. Khodayar, S. Mohammadi, M. E. Khodayar, J. Wang, and G. Liu, "Convolutional graph autoencoder: A generative deep neural network for probabilistic spatio-temporal solar irradiance forecasting," IEEE Trans. Sustain. Energy, vol. 11, no. 2, pp. 571583, Apr. 2020, doi: 10.1109/TSTE.2019.2897688.

[51] C. Huang, Z. Zhao, L. Wang, Z. Zhang, and X. Luo, "Point and interval forecasting of solar irradiance with an active Gaussian process,” IET Renew. Power Gener., vol. 14, no. 6, pp. 1020-1030, Apr. 2020, doi: 10.1049/IET-RPG.2019.0769.

[52] Q. Huang and S. Wei, "Improved quantile convolutional neural network with two-stage training for daily-ahead probabilistic forecasting of photovoltaic power," Energy Convers. Manag., vol. 220, p. 113085, Sep. 2020, doi: 10.1016/J.ENCONMAN.2020.113085.

[53] F. von Loeper, P. Schaumann, M. de Langlard, R. Hess, R. Bäsmann, and V. Schmidt, "Probabilistic prediction of solar power supply to distribution networks, using forecasts of global horizontal irradiation," Sol. Energy, vol. 203, pp. 145-156, Jun. 2020, doi: 10.1016/J.SOLENER.2020.04.001.

[54] D. van der Meer, D. Yang, J. Widén, and J. Munkhammar, "Clearsky index space-time trajectories from probabilistic solar forecasts: Comparing promising copulas," J. Renew. Sustain. Energy, vol. 12, no. 2, p. 026102, Apr. 2020, doi: 10.1063/1.5140604.

[55] R. Alonso-Suárez, M. David, V. Branco, and P. Lauret, "Intra-day solar probabilistic forecasts including local short-term variability and satellite information,” Renew. Energy, vol. 158, pp. 554-573, Oct. 2020, doi: 10.1016/J.RENENE.2020.05.046. 
[56] H. Quan and D. Yang, "Probabilistic solar irradiance transposition models," Renew. Sustain. Energy Rev., vol. 125, p. 109814, Jun. 2020, doi: 10.1016/J.RSER.2020.109814.

[57] G. M. Yagli, D. Yang, and D. Srinivasan, "Ensemble solar forecasting using data-driven models with probabilistic postprocessing through GAMLSS," Sol. Energy, vol. 208, pp. 612-622, Sep. 2020, doi: 10.1016/J.SOLENER.2020.07.040.

[58] H. Panamtash, Q. Zhou, T. Hong, Z. Qu, and K. O. Davis, "A copula-based Bayesian method for probabilistic solar power forecasting," Sol. Energy, vol. 196, pp. 336-345, Jan. 2020, doi: 10.1016/J.SOLENER.2019.11.079.

[59] M. Sun, C. Feng, and J. Zhang, "Probabilistic solar power forecasting based on weather scenario generation," Appl. Energy, vol. 266, p. 114823, May 2020, doi: 10.1016/J.APENERGY.2020.114823.

[60] K. Doubleday, V. Van Scyoc Hernandez, and B. M. Hodge, "Benchmark probabilistic solar forecasts: Characteristics and recommendations," Sol. Energy, vol. 206, pp. 52-67, Aug. 2020, doi: 10.1016/J.SOLENER.2020.05.051.

[61] H. He, N. Lu, Y. Jie, B. Chen, and R. Jiao, "Probabilistic solar irradiance forecasting via a deep learning-based hybrid approach," IEEJ Trans. Electr. Electron. Eng., vol. 15, no. 11, pp. 1604-1612, Nov. 2020, doi: 10.1002/TEE.23231.

[62] W. Liu and Y. Xu, "Randomised learning-based hybrid ensemble model for probabilistic forecasting of PV power generation," IET Gener. Transm. Distrib., vol. 14, no. 24, pp. 5816-5822, Dec. 2020, doi: 10.1049/IET-GTD.2020.0625.

[63] J. Le Gal La Salle, J. Badosa, M. David, P. Pinson, and P. Lauret, "Added-value of ensemble prediction system on the quality of solar irradiance probabilistic forecasts," Renew. Energy, vol. 162, pp. 1321-1339, Dec. 2020, doi: 10.1016/J.RENENE.2020.07.042.

[64] J. Munkhammar, D. van der Meer, and J. Widén, "Probabilistic forecasting of high-resolution clear-sky index time-series using a Markov-chain mixture distribution model," Sol. Energy, vol. 184, pp. 688-695, May 2019, doi: 10.1016/j.solener.2019.04.014.

[65] B. Chen and J. Li, "Combined probabilistic forecasting method for photovoltaic power using an improved Markov chain," IET Gener. Transm. Distrib., vol. 13 , no. 19 , pp. 4364-4373, Oct. 2019, doi: 10.1049/IET-GTD.2018.6860.

[66] K. Bakker, K. Whan, W. Knap, and M. Schmeits, "Comparison of statistical post-processing methods for probabilistic NWP forecasts of solar radiation," Sol. Energy, vol. 191, pp. 138-150, Oct. 2019, doi: 10.1016/j.solener.2019.08.044.

[67] M. Abuella and B. Chowdhury, "Forecasting of solar power ramp events: A post-processing approach," Renew. Energy, pp. 13801392, Apr. 2019, doi: 10.1016/j.renene.2018.09.005.

[68] C. A. Gueymard, "A review of validation methodologies and statistical performance indicators for modeled solar radiation data: Towards a better bankability of solar projects," Renew. Sustain. Energy Rev., vol. 39, pp. 1024-1034, Nov. 2014, doi: 10.1016/J.RSER.2014.07.117.

[69] D. Yang, J. Kleissl, C. A. Gueymard, H. T. C. Pedro and C. F. M. Coimbra, "Histoy and trends in solar irradiance and PV power forecasting: A preliminary assesment and review using text mining," Solar Energy., vol. 168, pp. 60-101, July 2018, doi: 10.1016/j.solener.2017.11.023.

[70] RK Sahu, B. Shaw, JR Nayak and Shashikant, "Short/medium term solar power forecasting of Chhattisgarh state of India using modified TLBO optimized ELM," Energy Sci. \& Tech., an international journal., vol. 24, no. 5, pp. 1180-1200, Oct. 2021, doi: 10.1016/j.jestch.2021.02.016 